\title{
THERAPY
}

\section{Silencing inhibitors of bone formation}

A key challenge in the clinical application of therapies based on small interfering RNAs (siRNAs) is a suitable vehicle for delivery, enabling direct targeting of the gene of interest whilst avoiding off-target effects. Now, a study published in Nature Medicine showcases a new liposomebased siRNA delivery system-dioleoyl trimethylammonium propane (DOTAP)based cationic liposomes attached to a (AspSerSer) ${ }_{6}$ repeat-that leads to the preferential accumulation of therapeutic siRNA at bone-formation surfaces and, ultimately, boosts bone formation. Will this new liposome delivery system for siRNAs silence the critics of RNA interference (RNAi)-based therapy for musculoskeletal disorders?

PLEKHO1 (also known as CKIP1) has been shown previously to be a suppressor of bone formation. Zhang and colleagues, therefore, reasoned that silencing this gene in osteoprogenitor cells could promote bone formation and might be useful as a treatment for osteoporosis. But how to deliver this potentially therapeutic siRNA into osteogenic-lineage cells?

Liposomes have emerged as one such delivery method for siRNAs. Thus, in this study, Plekho1 siRNA was encapsulated in DOTAP-derived cationic liposomes, which were then linked to a (AspSerSer) ${ }_{6}$ moiety in an attempt to preferentially anchor and target the siRNA to the boneformation surface; (AspSerSer) ${ }_{6}$ has high affinity to the small, randomly orientated hydroxyapatite crystals in osteoblastcovered bone-formation surfaces.

Zhang et al. found that systemic delivery of (AspSerSer) ${ }_{6}$-liposome Plekho1 siRNA in rats led to the enrichment of these siRNAs in osteogenic cells. Moreover, this approach promoted bone formation (without affecting bone resorption) and increased bone mass in healthy and osteoporotic rats.

"This delivery system could establish the foundation for translating RNAi-based therapies from basic science to clinical applications in the musculoskeletal field," write the study authors, who now plan to validate their findings in other animal models and cell systems, and to use their (AspSerSer) -liposome delivery system to target other proteins in vivo.

"An important drawback linked to the in vivo use of liposomes is toxicity, especially when repeated administrations are required for sustained RNAi activity," cautions Florence Apparailly (INSERM, University Hospital of Montpellier, France), who was not involved in the study. She adds that more work is needed

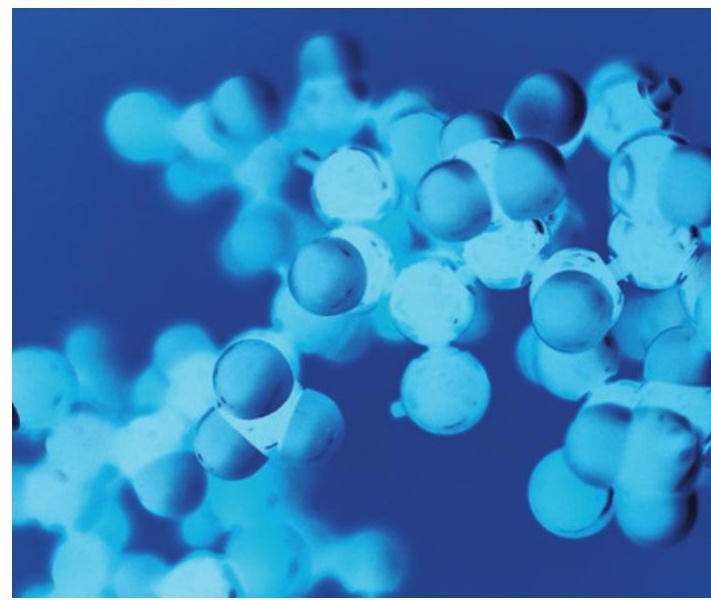

before these findings can be translated to the clinic: off-target effects of this new delivery system should be investigated; as should how long the silencing effect lasts; and in vivo experiments in humanized mouse models are needed to assess whether such a strategy works to deliver therapeutic siRNA to human osteoblast precursors. It is too soon to tell whether we will see the dawn of siRNA-based therapy for rheumatic diseases.

\section{Katrina Ray}

Original article Zhang, G. et al. A delivery system targeting bone formation surfaces to facilitate RNAi-based anabolic therapy. Nat. Med. 18, 307-314 (2012)

Further reading Whitehead, K. A. et al. Knocking down barriers: advance in siRNA delivery. Nat. Rev. Drug Discov. 8 129-138 (2009) 